\title{
Data Management of Age Determinations in (Pre-/Proto-)History
}

\author{
Yannic Ole KROPP ${ }^{\mathrm{a}, 1}$ and Bernhard THALHEIM ${ }^{\mathrm{a}, 2}$ \\ ${ }^{a}$ Christian Albrechts University Kiel, Department of Computer Science, D-24098 Kiel, \\ Germany
}

\begin{abstract}
This paper deals with the comparison and management of (heterogeneous) temporal datings in pre- and protohistory. It will present a first draft of a conceptual model for the description of the most common types of scales used in this context. The aim is to enable a system to compare objects according to their dating, regardless of the used method and scale. Thus temporally relevant objects can be selected by a query and do not need to be selected manually by an expert. Especially for larger data sets, automated computations, integrity checks, temporal reasoning or pattern mining this is beneficial.
\end{abstract}

Keywords. conceptual modelling, archaeological dating, data selection, data filtering, data management, reasoning

\section{Introduction}

Spatio-temporal analyses are an important tool in archaeology. ${ }^{3}$ Especially when examining transformations and processes of change the differences in spatial distributions over time are of high value. In this (short) paper we will focus on the temporal information needed to perform such analyses and deal with the task of managing data concerning archaeological datings.

Determining the age of archaeological objects is no uniform act. Depending on criteria like material, state of preservation, object-type, available funding and expertise different methods are applicable/available for the temporal classification. In effect the data is diverse regarding precision, accuracy, and robustness. Especially when the results of age determination map to different scales the comparison of datings is not trivial. Selecting adequate (data-)objects by their dating is therefore often done manually ${ }^{4}$ and thus time consuming. We want to ease this process by designing a (conceptual) model which enables the systematic description of 'temporal-dating-scales ${ }^{3}$ and their interlinkage in order to use these information for an automated comparison of dating instances regardless of the used scale.

For sure this approach cannot create more precision than given by the scales/methods and

\footnotetext{
${ }^{1}$ yok@informatik.uni-kiel.de

2 thalheim@is.informatik.uni-kiel.de

${ }^{3}$ see for example [5], [4], [6]

${ }^{4}$ Even when a database is used, has the 'allowed' time frame to be defined (manually) for each scale.

${ }^{5}$ We will explicitly not compare or rate the methods, but try to match the scales.
} 
thus is itself often vague and unprecise. But this is typical for this context. An archaeological dating is more or less an 'educated guess' and just possible to a significant degree of (statistical) probability. ${ }^{6}$ In addition datings often refer to phases or periods and not to a single point in time. For example do buildings have a certain time between construction and destruction during which they just exist. Typically the datings consist of ranges which might span several years. These ranges can be cultural epochs like 'neolithic', but may also occur as variance in scientific measurements. ${ }^{8}$ Also the transitions between temporal phases are in most cases no sharp events, but fuzzy, stepwise and procedural. ${ }^{9}$ We are aware of those (and more) effects which make the comparison of datings not very exact. Anyhow this challenge is normal in the given context and this is the kind of data which is available. When dealing with processes and objects from 10.000 (or more) years ago a certain fuzziness is unavoidable (not only in regard to dating). ${ }^{10} \mathrm{We}$ thus do not aim for a full interoperability of dating-scales, but for enabling basic ordinal operations. ${ }^{11}$ Primary this includes just the binary operations 'equals' $(=, \neq)$ and

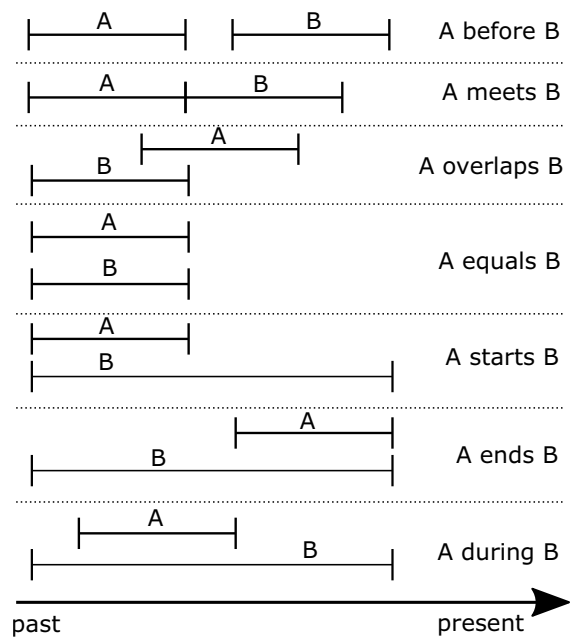

Figure 1. Possible relations between temporal periods according to [12]

'younger/older' $(<,>)$, but by representing periods by start and end times the basic relations of periods can also be used. Figure 1 shows these according to [12]. ${ }^{12}$

In effect the automated cross-scale comparison of datings won't be precise and exact, but will be sufficient for the purpose of identifying an initial set of potentially adequate objects by their dating. We will approach this objective by firstly examining and classi-

\footnotetext{
${ }^{6}$ The data is probabilistic but mostly not as sophisticated as in the probabilistic databases described in [14].

${ }^{7}$ part of the stone age

${ }^{8}$ The 'hallstatt plateau' is a famous example for a systematic variance up to 400 years in the radiocarbon dating method.

${ }^{9}$ 'Unfortunately' are often these transition phases the times which are scientifically most interesting.

${ }^{10}$ Archaeologists have certain methods to cope with it, see for example [11] or [10].

${ }^{11}$ For more information on ordinal scales (and other scales) see [13].

${ }^{12}$ As the datings are often not precise and a bit fuzzy, the relations which require an exact congruence in one point (meets, starts, ends, and maybe even equals) will probably in most cases result in FALSE, but especially the relations overlaps and during are very useful.
} 
fying the most common dating-scales in archaeology (section 2). ${ }^{13}$ Afterwards we will present a first draft of a conceptual model for the management of the dating-scales and actual dating-instances in section 3. Hereby we keep in mind, that the model shall enable ordinal comparisons of datings, regardless of the used scale. Finally section 4 will conclude our paper.

Remark: A previous version of this paper has been published under the title 'Modelling Temporal Scales in (Pre-/Proto-)History' [9].

\section{Scales of Dating}

Temporal datings in archaeology are not only performed by archaeologists, but for example also by experts in botany, (geo-)physics, chemistry, biology/anthropology, climate, etc. For example belongs the popular radiocarbon dating-method to the academic field of chemistry. Depending on the objects and the material which shall be dated the applicable methods vary. The mentioned radiocarbon dating-method is for example only applicable to organic material. Anyhow the variety of methods is not the topic of this paper. We will focus on the dating-instances which result from applying the methods and take a look on the used scales. ${ }^{14}$

Traditionally there are two major types of datings in archaeology. On the one hand $a b$ solute datings which refer to absolute points in time and on the other hand relative datings which refer to some event or object which does not necessarily has to be dated absolute. ${ }^{15}$ As we deal with the scales we will additionally differentiate between textual (mostly ordinal) scales and numeric/quantifiable (mostly interval or ratio) scales.

\section{1. absolute numeric dating}

Absolute numeric datings are typically the ones which also 'normal' people understand easily. Basic example for such a scale would be the Gregorian calendar and datings like $10.000 B C$ or $1066 A D$. A more 'archaeological' example are calibrated radiocarbon dates basing on the analysis of ${ }^{14} C$-isotopes. These give the age of objects in (ranges of) years before present $(B P) .{ }^{16}$ Also (calibrated) dates computed by using other isotopes fit in this category.

In general do scales of this category have two main features:

- a fixed global reference point, often given by a specific (gregorian) year

- a steady numeric unit of measurement, like (earth-)years, enabling comparisons of ratio

\footnotetext{
${ }^{13}$ As we are in involved in the research project $C R C 1266$, which explores transformations in pre- and protohistory, most of the presented scales are from that context.

${ }^{14}$ The information about the used methods are still valuable metadata and should not be disposed.

${ }^{15}$ Typically absolute datings are done by laboratories and are costly in terms of money and time and are thus not performed for all objects of an excavation. In addition is the (relative) on-site chronology often more important than the absolute dating of single objects (at least when dealing with just one/few site(s)).

16 'present' is in most cases assumed with the (gregorian) year 1950
} 


\section{2. relative numeric dating}

Relative numeric datings do also have a steady numeric unit of measurement, but lack the globally fixed reference point. Examples are:

- dendrochronology (tree-ring dating) - As a tree at average gains one growth-ring per year and the pattern of the rings is roughly the same for all trees originating from the same region ${ }^{17}$ it is possible to compare the difference in age of wooden objects in years/seasons using these information.

- laminated sediments - Under specific conditions the seasonal (organic) sediments (leaves, pollen, etc.) are visible in the soil of (former) lakes as single layers. It is possible to count the seasons/years between two layers.

- written records - In some cases ancient texts exist which describe events and their dating by using other events. Thus statements of the form 'event A took place 14 years after event B' are used for temporal information.

In general this type of scaling provides a chronology and a quantified temporal sequence, but no fixed reference on an absolute scale. Still this kind of scale might be mapped to an absolute scale if some of its objects are dated absolute. But the precision of absolute dating is often worse than the relative connections and thus the relative information stay highly valuable.

\section{3. absolute textual dating}

Absolute textual scales refer to datings which use labels that are seen as synonyms for (more or less) specific absolute time periods. These temporal periods do not need to be equally long or uniform. In particular these scales mostly refer to cultural epochs like Neolithic or Iron Age. But also climatic/geological periods like Holocene or Boreal fit to this category, as well as the dynasties of ancient Egypt.

While climatic and geological periods are mainly global phenomena and the egyptian dynasties mainly concern Egypt, do cultural epochs have a strong spatial component. Actually they are not datings per se but describe a certain state of society and technology. The Neolithic for example is defined by the presence of settlements, agriculture, husbandry, ceramics and (flint-)tools. This cultural state has been reached in different regions at different (absolute) times. Thus 'Neolithic in Germany' is not equivalent to 'Neolithic in Japan'. Also the duration of such phases differs depending on the regions. In effect there is not one global scale for cultural epochs, but multiple scales for the different regions. Although there are some general patterns and hierarchies in the sequence of the epochs, in some regions certain epochs are skipped or left out. Figure 2 shows an excerpt of an overview about differing durations of cultural epochs within Germany.

In summary this kind of scales can be characterised as follows:

(hierarchical) sequence of textual values which are used as synonyms for absolute time periods, in some cases (especially cultural epochs) having a scope on specific spatial regions

\footnotetext{
${ }^{17}$ same seasonal weather conditions etc.
} 


\section{Schleswig Holstein}

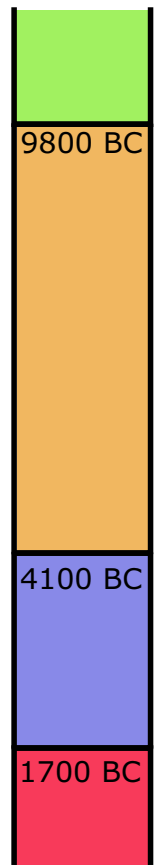

Bavaria

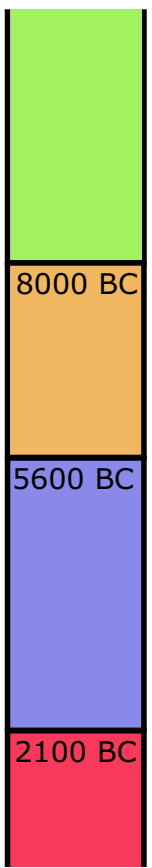

Saarland

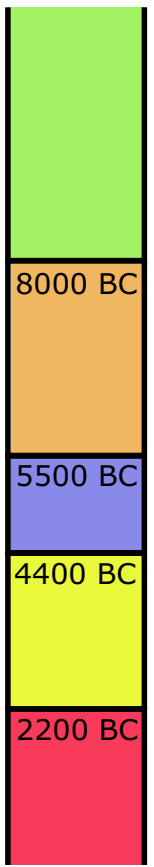

Paleolithic Mesolithic Neolithic Copper Age Bronze Age

Figure 2. differing durations of cultural epochs in three federal states of Germany - data taken from [7]

\section{4. relative textual dating}

Relative textual dates have neither a fixed global reference point nor a steady numeric unit of measurement. They consist of qualitative ordinal relations between objects, events, etc. and are often basing on common sense and heuristics. Some examples:

- These artefacts originate from the same grave, they were probably deposited at the same time.

- This coin/text mentions a famous person, it must have been created after or while that person lived.

- Artefact A was (three layers) deeper in the ground than artefact B. A must be older than $B$.

Even if a referred object/event can be dated absolute ${ }^{18}$ there is just an indirect transitive connection to an absolute scale for the remaining objects/events, but no actual absolute dating for them. So this type of scale is more useful for (relative) chronologies and to determine ages when something was (definitely) not existing.

\footnotetext{
${ }^{18}$ If for example tephra is found within a (geological) layer it is mostly possible to determine the exact (dated) volcanic eruption. See [15].
} 


\section{Handling different 'temporal' Datings}

As section 2 described, are the common ways of temporal dating in archaeology not always equivalent, but might refer to different types of scales. In order to enable (computer) systems to compare 'incongruent' datings we use a straightforward approach:

- describe scales in a conceptual model

- add used scale to every dating instance

Figure 3 shows our initial draft of the conceptual model for temporal datings and datingscales. Basically the model contains elements for each type of scaling which was described in the previous section. The absolute scales are backed with information about

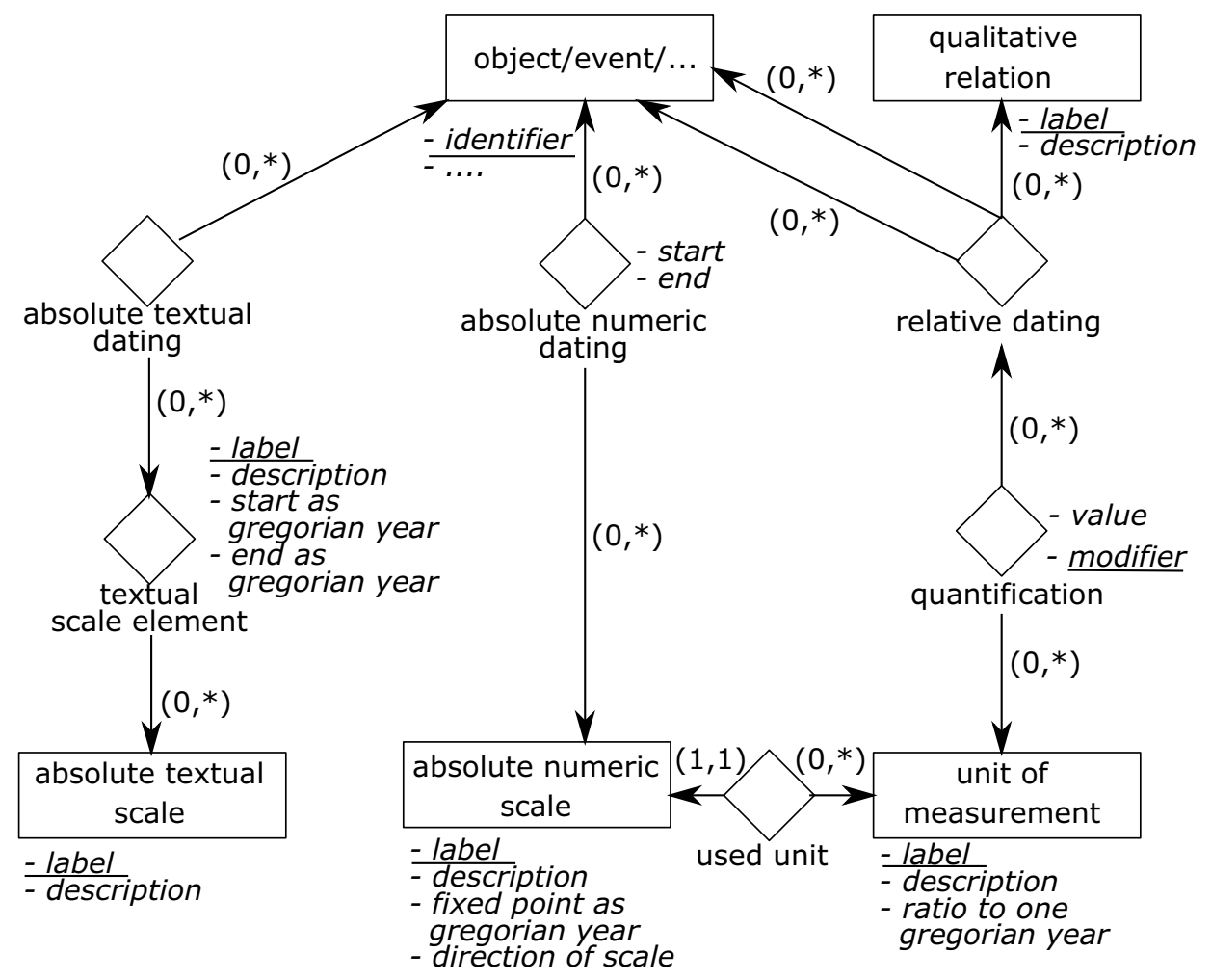

Figure 3. draft of a conceptual model for temporal datings and dating-scales

their orientation in the gregorian calendar. ${ }^{19}$ This information can be used (by automated functions) to check questions like:

Is 'Neolithic' on the northern-Europe scale the same like 'Neolithic' on the Mediterranean scale?

Is 4000 BC within the range of 'Neolithic' on the Mediterranean scale?

Is $4000 \mathrm{BC}$ the same as $6050 \mathrm{BP}$ ?

etc.

${ }^{19}$ having (sun-)years as the basic unit and a fixed starting point 2020 years ago 
Data of relative scales can be represented via pairs of objects ${ }^{20}$ and their qualitative relation $^{21}$. These basic relative information can be backed with quantitative information specifying the relation. ${ }^{22}$ This specification can again be enhanced by modifiers like 'exactly', 'at least', 'at most', etc.

In general the comparison of just absolute datings is quite unproblematic having the reference to the gregorian calendar as a 'mediating' scale. The comparison of relative datings or a relative dating and an absolute dating is more interesting. Especially when dealing with data from just one excavation-site, most of the objects will probably be initially dated with the same (unprecise) cultural period. ${ }^{23}$ In such a context the relative relations of the artefacts and relative temporal sequences are highly valuable. Artificial phases or concrete events are used as reference to gain a temporal order. By explicitly considering such relations in the conceptual model we are not only able to store these information, but also to use them for (automated) logical reasoning. Some examples:

- If $B$ is older than $A$ and $C$ is older than $B$ then must $C$ be older than $A$ and the sequence must be $C-B-A$. (due to transitivity of time)

- If $A$ is older than $B$ and $C$ is older than $B$, it is unclear if the sequence is $A-C-B, C$ $A-B$, or even $(A=C)-B$, but other sequences like $A-B-C, C-B-A$, etc. are definitely incorrect.

The ordinal position of two objects can apparently also be derived from absolute datings. And if an object is dated on more than one scale (especially on absolute and relative scales) objects which are (only) dated on different types of scales can (sometimes) be compared:

- $A$ is dated $4000 B C$ and $C$ is dated $4500 B C$ and $B$ is older than $C$ $\Longrightarrow B$ is (at least 500 years) older than $A$

But the direct temporal comparison of two objects is not always possible or (logically) derivable. If in the last example ' $C$ would have been older than $B$ ' the ordinal relation of $A$ and $C$ could not have been computed. ${ }^{24}$ Also the ordinal position of two objects which are both just dated by 'Mediterranean-Neolithic' is not clear, as this dating refers to a fairly long period.

Anyhow the result 'no ordinal relation is available/derivable' (due to lack of information) is acceptable, as an automated system will probably find this result faster than a person would and thus the work of archaeologists will still be eased. In addition the structure allows to (automatically) detect logical errors like circles, inconsistencies, contradictory values, etc. and to compute/check (feasible) chronological/ordinal sequences. ${ }^{25}$

\footnotetext{
${ }^{20}$ or events, etc.

21 'older', 'contemporary', etc.

${ }^{22}$ e.g. How many years older?

23 'This site, including all artefacts, is Neolithic.'

${ }^{24}$ At least if the relative relation of $A$ and $C$ wasn't quantified, like in ' $C$ is 30 years older than $B$ '.

${ }^{25}$ To perform this tasks the available ordinal information about objects can for example be viewed as mathematical transitive relations or directed graphs.
} 


\section{Conclusion \& Outlook}

In this (short) paper we deal with temporal datings of objects/artefacts in an archaeological context. We do not address the general concept of time (see [3]) or archaeological questions like 'What time-span is relevant for which phenomena?' (see [1], [2]), but restrict ourself to pure age determination. We though do not address challenges ${ }^{26}$ of the process 'dating' and its methods ${ }^{27}$ itself, but focus on data, scales, and comparability of object-datings. ${ }^{28}$

We characterised the different types of dating scales by the dimensions absolute-relative and textual-numeric. In general these types of scales are at least ordinal, but especially some of the numeric scales have interval- or even ratio-level. We introduced a draft for a conceptual model which allows the description of different scales and the storage of corresponding dating instances within one system.

The structure does also have the potential for enabling automated ordinal comparison of objects (in respect to temporal chronology) basing on the available datings regardless of the actual scales. Even if the available information is not sufficient for a meaningful result, the outcome that there's a lack of information is all right. Without sufficient information also a human expert could not perform better. In addition does the structure have potential for automated detection of contradictions and inconsistencies and for feasibility-tests of chronological sequences.

In summary does this (short) paper present initial ideas for handling temporal information across scales and a draft for the conceptual model, but no existing system. The systematic definition of rules, algorithms, and functions for (ordinal) comparisons, automated deduction, logical reasoning, etc. is part of future work.

Acknowledgement: This research was performed in the framework of the CRC $1266^{29}$ 'Scales of Transformation - Human-Environmental Interaction in Prehistoric and Archaic Societies' which is funded by the Deutsche Forschungsgemeinschaft (DFG, German Research Foundation - Projektnummer 2901391021 - SFB 1266) ${ }^{30}$. We thank both institutions for enabling this work.

\section{References}

[1] Geoff Bailey. Concepts, time scales and explanations in economic prehistory. 'Economic Archaeology', British Archaeological Reports, pages 97-117, 1981.

[2] Geoff Bailey. Time perspectives, palimpsests and the archaeology of time. Journal of anthropological archaeology, 26(2):198-223, 2007.

[3] Geoffrey N Bailey. Concepts of time in Quaternary prehistory. Annual review of anthropology, 12(1):165-192, 1983.

[4] Douglas James Cowie. Method and theory of archaeological spatio-temporal analysis. PhD thesis, University of Southampton, 2018.

\footnotetext{
${ }^{26}$ age of object vs. age of material, different ages (of different parts) in one object, etc.

${ }^{27}$ The used method is a valuable metadatum, which should be captured in another system-component.

${ }^{28}$ Other temporal entities like time of excavation, age of data, etc. and can for example be handled by Kramers general time-component [8].

${ }^{29}$ http://www.sfb1266.uni-kiel.de/en/

${ }^{30} \mathrm{http} / / / \mathrm{www} . \mathrm{dfg} \cdot \mathrm{de} / \mathrm{en} / \mathrm{index} . j \mathrm{sp}$
} 
[5] Centre for Baltic and Scandinavian Archaeology (ZBSA). EPHA - European prehistoric and historic atlas. http://www.zbsa.eu/zbsa/publikationen/open-access-datenmaterial/epha-european-prehistoric-andhistoric-atlas/.

[6] Daniel Knitter, Jan Piet Brozio, Wolfgang Hamer, Rainer Duttmann, Johannes Müller, and Oliver Nakoinz. Transformations and site locations from a landscape archaeological perspective: The case of Neolithic Wagrien, Schleswig-Holstein, Germany. Land, 8(4):68, 2019.

[7] Kommission Archäologie und Informationssysteme des Verbandes der Landesarchäologen (AG 'Thesauri'). Der 'Zeitstrahl' - Archäologische Datierungen in Deutschland. http://www.landesarchaeologen.de/verband/kommissionen/archaeologie-und-informationssysteme/, 2014.

[8] Frank Kramer. Ein allgemeiner Ansatz zur Metadaten-Verwaltung. Logos Verlag Berlin GmbH, 2019.

[9] Yannic Ole Kropp and Bernhard Thalheim. Modelling temporal scales in (pre-/proto-)history. In Proceedings of the 30th International Conference on Information Modelling and Knowledge Bases (EJC 2020), number 2020/1 in Kiel Computer Science Series, pages 192-199. Department of Computer Science, CAU Kiel, 2020.

[10] Doris Mischka. Aoristische Analyse in der Archäologie. Archäologische Informationen, 27(2):233-243, 2004.

[11] Oliver Nakoinz. Datierungskodierung und chronologische Inferenz-Techniken zum Umgang mit unscharfen chronologischen Informationen. Praehistorische Zeitschrift, 87(1):189-207, 2012.

[12] Richard T Snodgrass. Developing time-oriented database applications in SQL. The Morgan Kaufmann series in data management series, 2000.

[13] S. S. Stevens. On the Theory of Scales of Measurement. Science, 103 2684:677-80, 1946.

[14] Dan Suciu, Dan Olteanu, Christopher Ré, and Christoph Koch. Probabilistic databases. Synthesis lectures on data management, 3(2):1-180, 2011.

[15] Robert Walter. Potassium-Argon/Argon-Argon Dating Methods, volume 2, pages 97-126. 011997. 